$$
2
$$

Ästhetische Erfahrung und gesellschaftliches System 
Helmut Pfotenhauer

\section{Ästhetische Erfahrung und gesellschaftliches System}

Untersuchungen zu Methodenproblemen einer materialistischen Literaturanalyse am Spätwerk Walter Benjamins

J. B. Metzlersche Verlagsbuchhandlung Stuttgart 
Die vorliegende Arbeit wurde von der Philosophischen Fakultät der FriedrichAlexander-Universität Erlangen-Nürnberg als Dissertation angenommen. Sie geht zurück auf Vorstudien zum selben Thema, die unter dem Titel »Die Aktualisierung vergangener ästhetischer Erfahrungen als Aufgabe und Problem einer materialistischen Literaturinterpretation. Untersuchungen zum Spätwerk Walter Benjamins" kürzlich als Beitrag zu dem Band "Literaturwissenschaft und Sozialwissenschaften 4", Stuttgart 1974, veröffentlicht wurden. Gegenüber dem Aufsatz wurden erhebliche Erweiterungen und Veränderungen vorgenommen.

Für Unterstützung der Arbeit und Kritik hat der Verfasser insbesondere Kurt Wölfel und Heinz Schlaffer zu danken.

D 29

ISBN 978-3-476-00303-4

ISBN 978-3-476-03045-0 (eBook)

DOI 10.1007/978-3-476-03045-0

(C) 1975 Springer-Verlag GmbH Deutschland

Ursprünglich erschienen bei J. B. Metzlersche Verlagsbuchhandlung

und Carl Ernst Poeschel Verlag GmbH in Stuttgart 1975 


\section{Inhalt}

Vorbemerkung .................... 1

I. Benjamins geschichtsphilosophische Überlegungen zur Aufgabenstellung materialistischer Interpretation . . . . . . . . . .

1. Stillstand und geschichtliche Kontinuität. Der historische Materialismus als Kritik bürgerlicher und vulgärmarxistischer Ge-

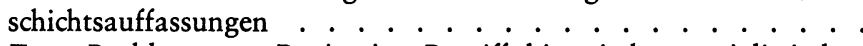

2. Zum Problem von Benjamins Begriff historisch-materialistischer Interpretation . . . . . . . . . . . 11

II. Über Charles Baudelaire . . . . . . . . . . . . . . . . . . 29

1. "Das Paris des Second Empire bei Baudelaire« . . . . . . . . 29

a) "Die Moderne" . . . . . . . . . . . . . . . . . 29

b) "Die Bohème«. Kritik und Bedeutung ästhetisierender Wirklichkeitsauffassung . . . . . . . . . . . . 37

c) Die Physiognomie des Flaneurs . . . . . . . . . . . . . 40

d) Die Fragwürdigkeit von Benjamins Interpretationsweise . . . 44

2. Die "Dialektik im Stillstand" . . . . . . . . . . . . . 59

a) Ästhetische Bildphantasie und Warenfetischismus . . . . . 59

b) Der Traum der Epoche und die Entwicklungstendenz zum Erwachen ............... . . 66 66

3. Strukturwandel der Erfahrung und Auraverlust der Kunst ... 70

a) Theorie des Erfahrungsverlusts . . . . . . . . . . 72

b) "Chokrezeption « und Wirkungsmöglichkeiten der Kunst im Zeitalter ihrer technischen Reproduzierbarkeit . . . . . . 76

4. Kulturindustrie und esoterische Kunst. $\mathrm{Zu}$ Adornos BenjaminKritik . . . . . . . . . . . . . . . . . 84

a) Heteronomie und Autonomie der Kunst in der Moderne . . . 84

b) Geschichtsphilosophie und Ästhetische Theorie . . . . . . . 89 Exkurs .................. . . 98

III. Zur Relativierung kunstphilosophischer Positionen. Benjamins Arbeiten über Brecht und Kafka . . . . . . . . . . . 103

1. Kommentare zu Brecht . . . . . . . . . . . . . . . . 103

a) Verwissenschaftlichung und Entprivatisierung der ästhetischen Erfahrungen . . . . . . . . . . . . . . . 104

b) $\mathrm{Zu}$ den historischen Voraussetzungen . . . . . . . . . 107

c) Intention und Implikationen der Kommentare zu Brecht . . . 112

2. Kafka . . . . . . . . . . . . . . . . 114 
Anmerkungen . . . . . . . . . . . . . . 120

Verzeichnis der zitierten Literatur . . . . . . . . . . . . 153

1. Primärliteratur . . . . . . . . . . . . . 153

2. Sekundärliteratur zu Benjamin und allgemeine Literatur ... 155

Namenregister. . . . . . . . . . . . . . . 160 


\section{Vorbemerkung}

Benjamins Philosophie und seine philosophisch geleiteten Interpretationen kultureller Phänomene stehen zur Marxschen Theorie der Gesellschaft in einem merkwürdigen Spannungsverhältnis. Die vorliegende Arbeit macht es sich zur Aufgabe, dem genauer nachzugehen. Uber Benjamin schreibt Adorno, daß er unbeirrt zu seinem Grundsatz gestanden habe, "die kleinste Zelle angeschauter Wirklichkeit wiege den Rest der ganzen Welt auf «. [1] Diese Bemerkung mag als vorläufige Charakterisierung gelten. Marx aber - so läßt sich wohl jenseits der Kontroverse über die Interpretation seiner Theorie sagen macht es sich zur Aufgabe, die der bürgerlichen Gesellschaft zugrunde liegenden allgemeingültigen Gesetzmäßigkeiten darzustellen. Die einzelne Erscheinung figuriert nur als Moment des sozialen Mechanismus. Als einzelne in ihrer unverwechselbaren Besonderheit gibt sie gerade keine Auskunft über den wahren Zustand der geschichtlichen Welt. Daraus könnte man folgern, daß nicht die »kleinste Zelle" selbst geschichtsphilosophisch bedeutsam sei, sondern eben jene allgemeinen Gesetze, die sie zusammen mit den anderen historischen Phänomenen bestimmen. Gleichwohl verstand sich Benjamin in den späteren Jahren seiner Produktion als historischer Materialist. Noch die "kleinste Zelle" müßte ihm also gerade im Sinne der Marxschen Gesellschaftstheorie in einer nicht vernachlässigbaren Weise aufschlußreich erschienen sein. Unmittelbar evident jedoch ist der Zusammenhang nicht, in den Benjamin beide Motive historischer Interpretation zu bringen versucht. Ihrer vorläufigen Kennzeichnung zufolge wirkt die Annahme ihrer Divergenz plausibler. Benjamins Bemühen, beides miteinander zu verbinden, ergibt denn auch den fragwürdigsten Aspekt seines Spätwerks. Wenn man dieses zu charakterisieren versucht, liegt es daher nahe, eine jener beiden Intentionen als die allein wesentliche hervorzuheben. Die jeweils andere wäre dann zu vernachlässigen oder sie wäre als Verirrung zu nehmen, welche der wahren Absicht des Autors nur abträgl:ch sein könne. Zweifellos bleibt aber eine solche Einstellung gegenüber Benjamins Spätwerk so lange unbefriedigend, so lange nicht geklärt ist, ob entgegen dem Augenschein nicht doch sachliche Gründe dafür sprechen, 
Benjamins Arbeiten als einen Vermittlungsversuch ernst zu nehmen zwischen der Theorie des historischen Materialismus und dem Interesse am Verständnis derjenigen Phänomene, deren aktuelle Bedeutung angesichts der allgemeinen Bewegungsgesetze gesellschaftlicher Reproduktion zunächst unabsehbar ist. Die vorliegende Untersuchung geht davon aus, daß es diese sachlichen Gründe gibt. Die Behauptung ist im weiteren zu belegen.

Eine ganz allgemeine Uberlegung zur Problemstellung gesellschaftstheoretisch fundierter Erklärung historischer Phänomene kann für die Legitimität dieser Annahme vorläufig einen Anhaltspunkt geben. Eine materialistisch orientierte Interpretation nämlich wird wie gesagt bestrebt sein, ihre Gegenstände aus den generellen, regelnden Prinzipien des gesellschaftlichen Lebens zu erklären und aufzuzeigen, daß sie aus sich selbst, etwa als autonome ästhetische Gebilde, nicht zureichend begriffen werden können. Eine solche Analyse muß aber immer in Rechnung stellen, die Besonderheit der untersuchten Erscheinungen zu verfehlen, das also, was diese von anderen unterscheidet, auch wenn sie in letzter Instanz alle gleichermaßen in Bezug zu setzen sind zu den zugrunde liegenden gesellschaftlichen Mechanismen. Dies gilt gerade für die als Beispiel genannten ästhetischen Produkte. Sie zeichnen sich durch die für sie konstitutive Distanz zur alltäglichen Lebenspraxis aus. Wenn nun auch diese Distanzierung selbst als gesellschaftlich veranlaßt begriffen werden kann, so ist Kunst doch nicht unmittelbar verständlich als Moment der gesellschaftlichen Reproduktion, ist nicht schlicht gleichzusetzen mit anderen Momenten, die in diesem sozialen Funktionszusammenhang bruchlos aufgehen. Darauf wird im folgenden zurückzukommen sein. Wenn dem aber so ist, wäre die Ableitung aus übergeordneten, generellen Prinzipien unzulänglich, weil sie ihren besonderen Gegenstand lediglich dem vorab schon gewußten Allgemeinen zuordnete. Sie unterstellte in sachlich fragwürdiger Weise, daß seine spezifischen Eigenschaften gegenüber seiner allgemeinen gesellschaftstheoretischen Charakteristik redundant seien. Will man dem begegnen, so ist es durchaus im Sinne einer auf die Marxsche Theorie ausgerichteten Interpretation, nach Möglichkeiten der Verbindung mit anderen Interpretationsvorhaben, wie dem angedeuteten von Benjamin, zu suchen. Benjamins Werk wird so gesehen eben deshalb interessant, weil es solche Zusammenhänge herzustellen beansprucht. Es ist eine These, die im Verlauf der Untersuchung erhärtet werden soll, daß diesem Anspruch gerecht zu werden geradezu als ein treibendes Motiv im Reflexionsprozeß verstanden werden kann, den Benjamins Spätwerk repräsentiert, und daß sich daraus zum einen Handhaben für dessen Verständnis ergeben, zum anderen aber auch einige Aufschlüsse über die Kompetenz und Grenzen einer materialistischen Interpretation ästhetischer Phäno- 
mene zu gewinnen sind. - Dabei soll nicht der Umstand aus dem Auge verloren werden, daß jene polaren interpretatorischen Intentionen in Benjamins intellektueller Biographie durchaus unterschiedlichen Ursprungs sind. Sie dürfen keineswegs nur als die Konsequenz aus systematischen Reflexionen auf die Leistungsfähigkeit marxistischer Kunsttheorie begriffen werden. Es wird zu berücksichtigen sein, daß von der Marxschen Theorie her gesehen eher apokryphe philosophische Motive für Benjamin von Bedeutung sind, die schließlich aber doch mit ihr in Verbindung gebracht werden. Zu klären ist, inwieweit diese Verbindung tatsächlich den Versuch einer reflektierten Vermittlung darstellt oder aber nur einen unvermittelten Bezug, welcher dann über das von Benjamin Explizierte hinaus soweit möglich in seinem Sinn zu rekonstruieren bzw. einer Kritik zu unterziehen wäre. In jedem Fall aber könnten sich in Anbetracht der oben skizzierten Fragestellung und der zu erwartenden Schwierigkeit ihrer Lösung gerade die von Benjamin angestellten oder veranlaßten unorthodoxen Überlegungen als fruchtbar erweisen.

Da die vorliegende Arbeit die Eigenart solcher Überlegungen Benjamins unter dem Gesichtspunkt jener allgemeinen Problematik untersuchen will, erscheint es nicht zweckdienlich, den Gang der Analyse allein auf die Abfolge der Entstehung von Benjamins Arbeiten auszurichten. Vielmehr soll gezeigt werden, wie weit es möglich ist, das Spätwerk Benjamins nach den Aspekten der genannten Problemstellung und den Stadien der Auseinandersetzung mit dieser Frage zu gruppieren. Zunächst jedoch ist die Problemstellung selbst in ihrer allgemeinen geschichtsphilosophischen und interpretationstheoretischen Fassung anhand verschiedener späterer Arbeiten Benjamins zu verdeutlichen. 